Pacific Journal of Mathematics

DEDEKIND DOMAINS: OVERRINGS AND SEMI-PRIM 


\title{
DEDEKIND DOMAINS: OVERRINGS AND SEMI-PRIME ELEMENTS
}

\section{LUTHER ClaborN}

\begin{abstract}
This paper develops two themes: (1) the relation of the class group of a Dedekind domain $A$ to that of an overring $B$ and (2) the question of finding a nonzero, nonunit element $x$ of a Dedekind domain $A$ such that $A / x A$ is regular. We obtain complete results in answer to the first question, giving a corollary concerning the realization of certain groups as class groups. We give various sufficient conditions in answer to the second question; some in terms of the class group, others concerning Dedekind domains which often arise in practice.
\end{abstract}

In $\S 1$ of the present paper, we study the class group of an overring $B$ of a Dedekind domain $A$ and determine its class group in terms of that of $A$. We generalize and also strengthen the results of $\S 1$ of an earlier article [1]. Combining several results, we obtain an interesting fact: if $G$ is the class group of a Dedekind domain and $G^{\prime}$ is a homorphic image of $G$, then $G^{\prime}$ is the class group of a suitable Dedekind domain.

Section 2 introduces the question of finding a nonunit $x$ in a Dedekind domain $A$ for which $A / x A$ is a direct sum of fields. Although we obtain no definitive result, various sufficient conditions are given. These require in part the developments of $\S 1$. We also give examples Dedekind domains with "pathological" class groups.

1. We state two well known propositions which we will need by way of background.

Proposition 1.1. Let $A$ be a Dedekind Domain with quotient field $F$. Let $B$ be a ring such that $A \subseteq B \subset F$. Then $B=\cap A_{p}$ over those prime ideals $P$ of $A$ for which $B \subseteq A_{p}$.

Proposition 1.2. Let $A$ be a Dedekind domain with quotient field $F$. Let $B$ be a ring such that $A \subseteq B \subset F$. Then $B$ is a Dedekind domain.

Proposition 1.3. Let $A$ be a Dedekind domain with quotient field $F$ and let $B$ be a ring such that $A \subseteq B \subset F$. The assignment $I \rightarrow I B$ is a homomorphism of the set of fractionary ideals of $A$ onto the set of fractionary ideals of $B$.

Received August 27, 1964. 
Proof. Let $Q$ be a prime ideal of $B$ and set $P=Q \cap A$. Then $P B=Q$. The inclusion $P B \subseteq Q$ is trivial, while $B_{Q}=A_{p}$ implies that $P B_{Q}=(P B) B_{Q}=Q B_{Q}$. This yields $P B=Q$ if we know that $P B$ is not contained in another prime ideal $Q^{\prime}$ of $B$. But then $Q^{\prime}$ would also lie over $P$, which is not the case by Prop. 1.1.

If $I$ is a fractionary ideal of $A$, then there is a $d \neq 0$ in $A$ such that $d I \subseteq A$. But then clearly $d(I B) \subseteq B$, so $I B$ is a fractionary ideal of $B$. The mapping is clearly a homomorphism for multiplication. To see that the mapping is onto, let $Q$ be a prime ideal of $B$. We have seen above that if $P=Q \cap A$, then $P B=Q$. Thus the mapping is onto the prime ideals of $B$, and these generate the group of fractionary ideals of $B$.

Corollary 1.4. Let $A$ be a Dedekind domain with quotient field $F$ and let $B$ be a ring such that $A \subseteq B \subset F$. The assignment $I \rightarrow I B$ of fractionary ideals of $A$ onto fractionary ideals of $B$ induces a homomorphism $\psi: \bar{I} \rightarrow \overline{I B}$ of the class group of $A$ onto that of $B$.

Proof. It is sufficient to note that if $I=x A$, then $I B=x B$.

Proposition 1.5. The kernel of $\psi$ is generated by all $\bar{P}_{\alpha}$, where $P_{\alpha}$ ranges over all prime ideals such that $P_{\alpha} B=B$.

Proof. Suppose $P_{\alpha} B=B$, and let $I$ be a fractionary ideal such that $\bar{I}=\bar{P}_{\alpha}$, i.e. $I=x P_{\alpha}$ for $x \in F$. Then $I B=x P_{\alpha} B=x B$, so $\overline{I B}$ is the identity.

Suppose now that $I$ is a fractionary ideal of $A$ such that $I B=y B$ for $y \in F$. Then $y I^{-1} B=B$, showing that $y I^{-1}$ is a product of primes $P_{\alpha}$ of $A$ for which $P_{\alpha} B=B$, and this establishes the assertion.

Corollary 1.6. Let $A$ be a Dedekind domain and $W=\left\{P_{\alpha}\right\}$ be a collection of primes such that $\left\{\bar{P}_{\alpha}\right\}$ does not generate the full class group of $A$. Then there are an infinite number of prime ideals of $A$ not in the set $\left\{P_{\alpha}\right\}$.

Proof. Let $B=\bigcap_{P \notin W} A_{p}$. By Proposition $1.5, B$ is not a principal ideal domain. Therefore there are an infinite number of prime ideals of $B$, hence an infinite number of prime ideals of $A$ which are not in $W$.

Corollary 1.7. Let $A$ be a Dedekind domain with class group 
G. Let $H$ be any subgroup of $G$. Then there is a Dedekind domain whose class group is $\mathrm{G} / \mathrm{H}$.

Proof. In [1], we constructed the Dedekind domain $A^{\prime}=A[X]_{S}$, where $S$ denotes the set of all monic polynomials of $A[X]$. We showed that $A$ has the same class group as $A[1$, Prop. 2.3] and also that $A$ has a prime ideal in every class of the class group [1, Cor. 2-5]. Identify $G$ and $H$ at the class group and a subgroup of the class group of $A^{\prime}$. For each class of $H$, choose a prime $P^{\prime}$ of $A^{\prime}$ in the given class. Let $W$ denote the set $\left\{P^{\prime}\right\}$ so chosen. Then $B=\bigcap_{Q \oplus W} A_{Q}$ has class group $G / H$ by Proposition 1.5.

2. Definition 2.1. Let $A$ be a Dedekind domain. An element $x$ of $A$ which is not zero and not a unit will be said to be semi-prime if $A / x A$ is a regular ring.

REMARK 2.2. This condition is equivalent to (1) $A / x A$ is a direct sum of fields, or (2) $x A$ is not contained in the square of any prime ideal of $A$.

In what follows, sufficient conditions will be given for $A$ to contain semi-prime elements. If $A$ has only a finite number of prime ideals, then $A$ is a principal domain and obviously $A$ contains semi-prime elements. This case ( $A$ has only a finite number of prime ideals) will be excluded from the developments which follow.

Proposition 2.3. If $A$ has a finite class group, then there are semi-prime elements in $A$.

Proof. Since we are assuming that $A$ has infinitely many prime ideals, there must be at least one class of the class group containing an infinite set $\left\{P_{i}\right\}$ of the prime ideals. If $n$ is the class number of $A$, then $P_{1} \cdots P_{n}$ must be principal, say $x A=P_{1} \cdots P_{n}$. $x$ is then a semi-prime element.

Proposition 2.4. Let $A$ be a Dedekind domain, and suppose that every class of the class group (except possibly the principal class) contains a prime ideal. Then $A$ contains a semi-prime element.

Proof. If $A$ is a principal ideal domain, then there is nothing to prove. Otherwise let $P$ be a nonprincipal prime ideal and let $Q$ be a prime in the class of $P^{-1}$. Then $P Q$ is principal, say $P Q=x A$, and $x$ will be semi-prime unless $P=Q$. We are therefore done unless every class has exponent 2 and there is only one prime in each class. 
Choose $P$ to represent one nonprincipal class and $Q$ to represent a different nonprincipal class. Choose a prime ideal $R$ in the class of $P Q$. Obviously $R \neq P, R \neq Q$, while $P Q R$ is principal. This gives a semi-prime element in $A$.

We can actually prove a little more.

Proposition 2.5. Let $A$ be a Dedekind domain, and suppose that for every prime ideal $P$ there is a prime of $A$ in the class of $P^{-1}$. Then $A$ contains a semi-prime element.

Proof. As in the proof Proposition 2.4 we may assume that every class has exponent 2 . The class group of $A$ may therefore be regarded as a vector space over the field with 2 elements. Since the prime ideals of $A$ generate the class group, we may choose a basis $\left\{\bar{P}_{\alpha}\right\}$ for the class group consisting of classes of prime ideals. Let $P$ be any prime ideal of $A$ and let $\bar{P}$ be its class. Let $\bar{P}=\bar{P}_{\alpha_{1}} \ldots \bar{P}_{\alpha_{k}}$ be its representation in terms of the given basis. Thus $P P_{\alpha_{1}} \cdots P_{\alpha_{k}}$ is principal and we get a semi-prime element unless $P$ is in the set $\left\{P_{\alpha}\right\}$. We may assume then that the set $\left\{P_{\alpha}\right\}$ contains all prime ideals of $A$. But this contradicts Corollary 1.6, and the proposition is established.

Before giving an example violating the hypothesis of Proposition 2.5, we present a lemma which will be useful in constructing such an example and in a later proof.

LEMMA 2.6. Let $F$ be a field of characteristic $p$ such that $\left[F^{1 / p}: F\right]=p$. Let $K$ be a separable extension of $F ;$ then $\left[K^{1 / p}: K\right]=p$.

Proof. Since $K$ is a separable extension of $F$, we have $K=F\left(K^{p}\right)$ [3, Thm. 8, p. 69]. Thus $K^{1 / p}=F^{1 / p}(K)$. But $F^{1 / p}$ and $K$ are linearly disjoint $\left[3\right.$, Thm. 35, p. 111], so we get $\left[K^{1 / p}: K\right]=\left[F^{1 / p}(K): K\right]=$ $\left[F^{1 / p}: F\right]=p$.

EXAMPLE 2.7. Let $F^{\prime}=Z / 3 Z(\alpha)$ where $Z$ denotes the integers and $a$ is indeterminant. Let $F$ be the separable closure of $F^{\prime}$ in its algebraic closure. By Lemma 2.6, $\left[F^{1 / 3}: F\right]=3$. Consider the integral closure $A$ of $F[X]$ in the field $F(X, Y)$, where $Y^{3}=a X^{3}+X$. It is not difficult to show by a direct computation that $A=F[X, Y]$, but it is easier to notice that since the matrix of partial derivatives of the equation $Y^{3}-a X^{3}-X$ has always rank $1, F[X, Y]$ is regular [2, Thm. 1. p. 201]. Over each prime ideal of $F[X]$ there lies only one prime ideal of $A$ and for the relative degree $f$ of the residue field and the ramification index $e$ we have $\mathrm{e}=3, f=1$ or $e=1, f=3[3$, 
Thm. 22, p. 289]. We show first that for all nonlinear prime elements of $F[X]$, we get $e=1, f=3$, so these remain principal. Let $Q$ be a prime ideal of $F[X]$ generated by a nonlinear element; $Q=X^{q}-t$, where $q$ is a power of 3 and $t \in F$. The residue field $F[X] /\left(X^{q}-t\right)$ is $F\left[t^{1 / q}\right]$, while the residue field relative to $A$ will be $F\left[t^{1 / q}, w\right]$, where $w^{3}=a t^{3 / q}+t^{1 / q}$. Since $\left[F^{1 / 3}: F\right]=3$, we have $F^{1 / 3} \subseteq F\left[t^{1 / q}\right]$, hence $a^{1 / 3} \in F\left[t^{1 / q}\right]$. Thus $a t^{3 / q}$ is a cube in $F\left[t^{1 / q}\right]$. But $t^{1 / q}$ is not a cube in $F\left[t^{1 / q}\right]$, so $\left[F\left[t^{1 / q}, w\right]: F\left[t^{1 / q}\right]\right]=3$. That is, $f=3, e=1$; thus we see that nonlinear prime elements of $F[X]$ remain prime in $A$.

For the linear primes $X-t, t \varepsilon F$, we get $e=1, f=3$ if $a t^{3}+t$ is not a cube in $F$, while $e=3, f=1$ if at $t^{3}+t$ is a cube in $F$. Certainly we have the latter case at least for $t=0$. Let $P$ be a prime ideal of $A$ lying over a linear prime ideal of $F[X]$ for which $e=3, f=1$. Then $P$ is not principal。 For if $P$ were principal, say $P=\left(c_{0}(X)+c_{1}(X) Y+c_{2}(X) Y^{2}\right)$ we would get

$$
P^{3}=\left(c_{0}^{3}(X)+c_{1}^{3}(X)\left(a X^{3}+X\right)+c_{2}^{3}(X)\left(a^{2} X^{6}+2 \alpha X^{4}+X^{2}\right) .\right.
$$

But $P^{3}=(x-t)$ for some $t \in F$. Comparing degrees and using the fact that $1, a, a^{2}$ are independent over $F^{3}$, we get a contradiction. Again let $P$ be such a prime and suppose that the class of $P^{2}$ (which is the clsss of $P^{-1}$ ) contains a prime $Q . Q$ is certainly not principal ; therefore $Q$ lies over a linear prime ideal of $F[X]$ and $e=3, f=1$ for $Q$. We also get that $P^{2} Q^{2}$ is principal, say

$$
P^{2} Q^{2}=\left(d_{0}(X)+d_{1}(X) Y+d_{2}(X) Y^{2}\right) \text {. }
$$

Cubing, we get

$$
\left(P^{3}\right)^{2}\left(Q^{3}\right)^{2}=\left(d_{0}^{3}(X)+d_{1}^{3}(X)\left(a X^{3}+X\right)+d_{2}^{3}(X)\left(a^{2} X^{6}+2 a X^{4}+X^{2}\right)\right) .
$$

On the left side of this equation we have a polynomial of degree 4 , while on the right we have a polynomial whose degree is divisible by 3 , a contradiction.

Proposition 2.8. Let $A$ be a principal ideal domain and let $K$ be a finite separable extension of the quotient field $F$ of $A$. Let $B$ be the integral closure of $A$ in $K$ and let $C$ be a ring such that $B \subseteq C \subset K$. Then $C$ contains a semi-prime element. ${ }^{1}$

Proof. There are only a finite number of prime ideals $Q_{1}, \cdots, Q_{k}$ of $B$ whose reduced ramification index is greater than 1 [3, Thm. 28, p. 302]. Let $P=\pi A$ be a prime ideal of $A$ not lying under any $Q_{1}, \cdots, Q_{k}$. Then $\pi B$ is a product of distinct primes and is a semi-

1 The referee has kindly pointed out that this Proposition (and thus the following) hold when $B$ is not necessarily integrally closed. 
prime element in $B . \quad \pi$ will also be a semi-prime element in $C$ unless all prime ideals of $B$ dividing $\pi$ generate $C$. The result now follows by Proposition 1.5 and Corollary 1.6.

Proposition 2.9. Let $A$ be the coordinate ring of an algebraic curve over a perfect ground field $F$. If $A$ is a Dedekind domain, then $A$ contains a semi-prime element.

Proof. $A=F\left[x_{1}, \cdots, x_{n}\right]$. Choose $X$ in $A$ such that $A$ is integral over $F[X]$; this is possible by [2, Thm. 1, p. 22]. Since $A$ is integrally closed in $K=F\left(x_{1}, \cdots, x_{n}\right), A$ is the integral closure of $F[X]$ in $K$. Let $K^{\prime}$ be the separable closure of $F(X)$ in $K$, and let $A^{\prime}$ be the integral closure of $F[X]$ in $K$. The conclusion holds for $A^{\prime}$ by Proposition 2.8.

Since $\left[F(X)^{1 / p}: F(X)\right]=p$, we have $\left[K^{1 / p}: K^{\prime}\right]=p$ by Lemma 2.6. $K$ is a purely inseparable extension of $K^{\prime}$, so we may break the extension from $K^{\prime}$ to $K$ into a chain of extensions each of which is pure inseparable of exponent $p$. This chain can only be

$$
K^{\prime}=K_{0} \subset K_{0}^{1 / p} \subset K_{0}^{1 / p^{2}} \subset \cdots \subset K_{0}^{1 / p m}=K .
$$

But then we have an isomorphism of $K$ onto $K^{\prime}$ given by $x \rightarrow x^{p m}$ which induces an isomorphim of $A$ onto $A^{\prime}$. Since $A^{\prime}$ contained semiprime elements, so does $A$.

\section{REFERENCES}

1. L. Claborn, Dedekind domains and rings of quotients, Pacific J. Math. 15 (1965), 59-64.

2. S. Lang, Introduction to Algebraic Geometry, New York, Interscience Publishers, Inc. (1958).

3. O. Zariski and P. Samuel, Commutative Algebra, Vol. I, Princeton, D. Van Nostrand Company (1958).

CORNELl COLLEge 


\section{PACIFIC JOURNAL OF MATHEMATICS}

\section{EDITORS}

\author{
H. Samelson \\ Stanford University \\ Stanford, California \\ R. M. Blumenthal \\ University of Washington \\ Seattle, Washington 98105
}

\author{
J. Dugundu \\ University of Southern California \\ Los Angeles, California 90007 \\ *Richard Arens \\ University of California \\ Los Angeles, California 90024
}

\section{ASSOCIATE EDITORS}
E. F. BeCKENBACH
B. H. NeUMANN
F. WOLF
K. YosidA

\section{SUPPORTING INSTITUTIONS}

\author{
UNIVERSITY OF BRITISH COLUMBIA \\ CALIFORNIA INSTITUTE OF TECHNOLOGY \\ UNIVERSITY OF CALIFORNIA \\ MONTANA STATE UNIVERSITY \\ UNIVERSITY OF NEVADA \\ NEW MEXICO STATE UNIVERSITY \\ OREGON STATE UNIVERSITY \\ UNIVERSITY OF OREGON \\ OSAKA UNIVERSITY \\ UNIVERSITY OF SOUTHERN CALIFORNIA
}

\author{
STANFORD UNIVERSITY \\ UNIVERSITY OF TOKYO \\ UNIVERSITY OF UTAH \\ WASHINGTON STATE UNIVERSITY \\ UNIVERSITY OF WASHINGTON \\ * * * * \\ AMERICAN MATHEMATICAL SOCIETY \\ CALIFORNIA RESEARCH CORPORATION \\ SPACE TECHNOLOGY LABORATORIES \\ NAVAL ORDNANCE TEST STATION
}

Mathematical papers intended for publication in the Pacific Journal of Mathematics should by typewritten (double spaced). The first paragraph or two must be capable of being used separately as a synopsis of the entire paper. It should not contain references to the bibliography. No separate author's resumé is required. Manuscripts may be sent to any one of the four editors. All other communications to the editors should be addressed to the managing editor, Richard Arens, at the University of California, Los Angeles, California 90024.

50 reprints per author of each article are furnished free of charge; additional copies may be obtained at cost in multiples of 50 .

The Pacific Journal of Mathematics is published quarterly, in March, June, September, and December. Effective with Volume 13 the price per volume (4 numbers) is $\$ 18.00$; single issues, $\$ 5.00$. Special price for current issues to individual faculty members of supporting institutions and to individual members of the American Mathematical Society: $\$ 8.00$ per volume; single issues $\$ 2.50$. Back numbers are available.

Subscriptions, orders for back numbers, and changes of address should be sent to Pacific Journal of Mathematics, 103 Highland Boulevard, Berkeley 8, California.

Printed at Kokusai Bunken Insatsusha (International Academic Printing Co., Ltd.), No. 6, 2-chome, Fujimi-cho, Chiyoda-ku, Tokyo, Japan.

PUBLISHED BY PACIFIC JOURNAL OF MATHEMATICS, A NON-PROFIT CORPORATION

The Supporting Institutions listed above contribute to the cost of publication of this Journal, but they are not owners or publishers and have no responsibility for its content or policies.

* Basil Gordon, Acting Managing Editor until February 1, 1966. 


\section{Pacific Journal of Mathematics}

\section{Vol. 15, No. $3 \quad$ November, 1965}

David R. Arterburn and Robert James Whitley, Projections in the space of

bounded linear operators .................................

Robert McCallum Blumenthal, Joram Lindenstrauss and Robert Ralph Phelps,

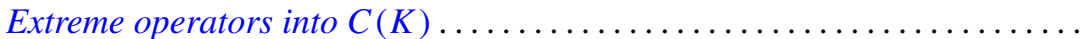

L. Carlitz, A note on multiple exponential sums ................... 757

Joseph A. Cima, A nonnormal Blaschke-quotient .................... 767

Paul Civin and Bertram Yood, Lie and Jordan structures in Banach algebras . . . 775

Luther Elic Claborn, Dedekind domains: Overrings and semi-prime

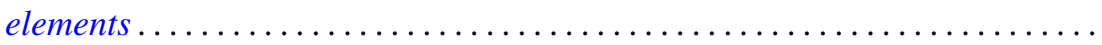

799

Luther Elic Claborn, Note generalizing a result of Samuel's .............. 805

George Bernard Dantzig, E. Eisenberg and Richard Warren Cottle, Symmetric

dual nonlinear programs ................................... 809

Philip J. Davis, Simple quadratures in the complex plane ............... 813

Edward Richard Fadell, On a coincidence theorem of F. B. Fuller ............ 825

Delbert Ray Fulkerson and Oliver Gross, Incidence matrices and interval

graphs ........................................ 835

Larry Charles Grove, Tensor products over $H^{*}$-algebras ..................

Deborah Tepper Haimo, $L^{2}$ expansions in terms of generalized heat polynomials

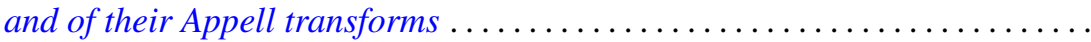

I. Martin (Irving) Isaacs and Donald Steven Passman, A chardcterization of groups in terms of the degrees of their characters ..........

Donald Gordon James, Integral invariants for vectors over local fields........ 905

Fred Krakowski, A remark on the lemma of Gauss ................... 917

Marvin David Marcus and H. Minc, A subdeterminant inequality ........... 921

Kevin Mor McCrimmon, Norms and noncommutative Jordan algebras ........ 925

Donald Earl Myers, Topologies for Laplace transform spaces ............... 957

Olav Njstad, On some classes of nearly open sets .................... 961

Milton Philip Olson, A characterization of conditional probability ........... 971

Barbara Osofsky, A counter-example to a lemma of Skornjakov .............. 985

Sidney Charles Port, Ratio limit theorems for Markov chains ............... 989

George A. Reid, A generalisation of $W^{*}$-algebras ...................... 1019

Robert Wells Ritchie, Classes of recursive functions based on Ackermann's

function ........................................... 1027

Thomas Lawrence Sherman, Properties of solutions of $n$th order linear

differential equations ........................................ 1045

Ernst Snapper, Inflation and deflation for all dimensions . .............. 1061

Kondagunta Sundaresan, On the strict and uniform convexity of certain Banach

spaces............................................. 1083

Frank J. Wagner, Maximal convex filters in a locally convex space .......... 1087

Joseph Albert Wolf, Translation-invariant function algebras on compact 